\title{
EDUCACIÓN SUPERIOR SOCIO-INCLUSIVA EN EL CONURBANO BONAERENSE: el caso de la Universidad Nacional de Hurlingham (UNaHur)
}

\author{
ENSINO SUPERIOR SOCIALMENTE INCLUSIVO NA GRANDE BUENOS AIRES: \\ o caso da Universidade Nacional de Hurlingham (UnaHur)
}

SOCIO-INCLUSIVE HIGHER EDUCATION IN GREATER BUENOS AIRES: the case of the National University of Hurlingham (UNaHur)

\author{
Roxana Regina Taranto Orilio ${ }^{1}$ \\ https://orcid.org/0000-0002-8771-6807 \\ Santiago Durante \\ https://orcid.org/0000-0003-0919-8183 \\ Jorge Alejandro Santos 3 \\ https://orcid.org/0000-0002-9081-5881
}

\begin{abstract}
Resumen
Este artículo tiene como objetivo exponer una experiencia de educación inclusiva en el contexto de la educación superior Argentina. El país tiene una tradición histórica en relación a la inclusión y el acceso a la educación superior. Sin embargo, la sola posibilidad de ingreso a la enseñanza universitaria no garantiza el acceso real de todas y todos. Hay desigualdades sociales, territoriales, de capital simbólico que hacen que, para algunos sectores sociales, el ingreso a este nivel educativo sea difícil incluso en un contexto de gratuidad y acceso irrestricto. En las últimas décadas se crearon universidades que por su ubicación territorial y su proyecto pedagógico contribuyen a mitigar estas
\end{abstract}

1 Licenciada em Historia-Universidad de Lujan. Especialista en Historia de la Pedagogía Latinoamericana. Profesora de la Universidad de Hurlingham, provincia de Buenos Aires, Argentina. E-mail: roxana taranto@hotmail.com

2 Doctor y Profesor en Letras UBA. Investigador y docente da Universidad Nacional de Hurlingham (UNaHur), Villa Tesei, Argentina. Becario postdoctoral (CONICET) en Lingüística UBA. Email: santiago.durante@unahur.edu.ar

3 DoctorenFilosofía por la Universidad de Buenos Aires (2009-2015 UBA). Investigador de la Unversidad Nacional de Hurlinhgam, Argentina. Profesor Colaborador del PPGE Unochapecó, Brasil. Profesor de Posgraduación en Filosofía, Facultad de Filosofía y Letras UBA. Profesor de Filosofía de la Ciencia en el Ciclo Básico Común UBA y en la Facultad de Ciencias Económicas de la Universidad de Lomas de Zamora. E-mail: jorgesantosuba@gmail.com

\section{Como referenciar este artigo:}

ORILIO, R. R. T.; DURANTE, S.; SANTOS, J. A. Educación superior socio-inclusiva en el conurbano bonaerense: el caso de la Universidad Nacional de Hurlingham (UNaHur). Revista Pedagógica, $v$. 23, p. 1-22, 2021. 
desigualdades. Entre ellas se encuentran las llamadas universidades del conurbano bonaerense de las cuales la UNaHur forma parte siendo la de creación más reciente. El trabajo expondrá la experiencia de esta nueva universidad, a partir de su inserción en la tradición histórica de las educación argentina y de su proyecto político-pedagógico inclusivo.

Palabras clave: universidad, inclusión social, conurbano, desigualdad, Hurlingham.

\title{
Resumo
}

Este artigo tem como objetivo expor uma experiência de educação social inclusiva no contexto do ensino superior na Argentina. O país tem uma tradição histórica em relação à inclusão e acesso ao ensino superior. No entanto, a mera possibilidade de ingressar no ensino universitário não garante acesso real a todos e todas. Existem desigualdades sociais, territoriais e simbólicas que dificultam a entrada nesse nível educacional para alguns setores sociais, mesmo em um contexto de acesso livre e irrestrito. Nas últimas décadas, foram criadas universidades que, devido à sua localização territorial e ao seu projeto pedagógico, contribuem para mitigar essas desigualdades. Entre elas estão as chamadas universidades dos subúrbios de Buenos Aires, das quais o UNaHur faz parte, sendo as mais recentes criadas. O trabalho expõe a experiência desta nova universidade, desde sua inserção na tradição histórica da educação argentina e seu projeto político-pedagógico inclusivo.

Palavras-chave: universidade, inclusão social, subúrbios, desigualdade, Hurlingham.

\begin{abstract}
This article aims to expose an experience of inclusive social education in the context of higher education in Argentina. The country has a historical tradition of inclusion and access to higher education. However, the mere possibility of entering university education does not guarantee real access to everyone. There are social, territorial, and symbolic inequalities that make entry to this educational level difficult for some social sectors, even in a context of free and unrestricted access. In the last decades, universities have been created that, due to their territorial location and their pedagogical project, contribute to mitigate these inequalities. Among them are the so-called universities of the Buenos Aires suburbs, of which UNaHur is part, being the most recently created. The paper exposes the experience of this new university, from its insertion in the historical tradition of Argentine education and its inclusive political-pedagogical project.
\end{abstract}

Keywords: university, social inclusion, suburbs, inequality, Hurlingham.

\section{INTRODUCCIÓN}

Este artículo tiene como objetivo exponer una la experiencia de educación inclusiva en el contexto de la educación superior de la Argentina. El predicado “inclusivo" aplicado a la educación puede entenderse de diferentes modos; en su sentido más general tiene que ver con una política educativa que facilita y promueve el ingreso y la permanencia en los distintos niveles educativos a grupos que han enfrentado históricamente dificultades para ingresar y permanecer en el sistema. Estas dificultades pueden originarse en diferentes 
factores: clase social, habilidades o dificultades físicas o cognitivas en el caso de personas con discapacidad, en diferencias culturales o étnicas, etc.

La educación superior argentina tiene una amplia tradición de inclusión y acceso a la educación superior con hitos como el movimiento reformista de 1918 para democratizar el acceso a la universidad y al saber científico, la gratuidad de la educación en 1949 y la democratización del acceso a partir del fin de la dictadura militar en 1983.

Sin embargo, la sola posibilidad de ingreso a la enseñanza universitaria no garantiza el acceso real de todas y todos a la educación superior. Hay desigualdades sociales, territoriales, de capital simbólico que hacen que para algunos sectores el ingreso a la educación superior sea difícil incluso en un contexto de gratuidad y acceso irrestricto como el argentino.

En distintos momentos históricos de nuestro país se crearon políticas e instituciones universitarias nuevas a fin de ampliar este acceso. El último período de creación de nuevas universidades con esta finalidad inclusiva es reciente, comienza a inicios de siglo y se detiene a finales de 2015. La última universidad creada es justamente la Universidad Nacional de Hurlingham (UNaHur) ubicada en el conurbano de la Ciudad de Buenos Aires y tiene como función y objetivo explícito contribuir al desarrollo local y nacional a través de la producción y distribución equitativa de conocimientos e innovaciones científicotecnológicas (UNAHUR, 2020). Esta institución se encuentra en la localidad de Villa Tesei, dentro del municipio de Hurlingham. Su ubicación territorial facilita el acceso real a la educación universitaria a una enorme población perteneciente, en general, a sectores populares. En un porcentaje muy elevado la matrícula de las universidades del conurbano, entre el 70 y el 95\% según las instituciones (MANGAS, ROVELLI, 2020) está formada por estudiantes que constituyen la primera generación de universitarios de sus familias, y muchos de ellos también, la primera generación que logró concluir los estudios secundarios. Pero la UNaHur además de su inserción territorial tiene un proyecto políticopedagógico inclusivo dirigido específicamente a facilitar el acceso y promover la permanencia de los estudiantes pertenecientes a esa población y que ha sido exitoso en su desempeño durante sus cinco años de existencia llegando a una matrícula que en el año 2020 supero los diez mil estudiantes. 
En este artículo expondremos el proyecto político-pedagógico inclusivo de la institución e ilustraremos sus resultados. A tal fin el artículo comenzará con la descripción del marco teórico y metodológico del estudio propuesto definiendo fundamentalmente la idea de educación inclusiva, continuará con una contextualización histórica del sistema universitario argentino centrado en explicar el surgimiento de las llamadas universidades del conurbano y finalmente se expondrá el proyecto inclusivo de la universidad y sus resultados.

\section{MARCO TEÓRICO-METODOLÓGICO}

\subsection{El concepto de educación inclusiva}

El concepto de educación inclusiva surge aproximadamente hace tres décadas asociado a la crítica del sistema de educación especial que restringía o vedaba el acceso de las personas con discapacidades físicas y/o a los distintos niveles del sistema educativo (RAMBLA et al, 2008). La UNESCO ha tenido un protagonismo importante en torno a este concepto acuñándolo y dándole relevancia a partir de la Declaración de Salamanca del año 1994, que ha sido posiblemente el documento internacional de mayor trascendencia en el campo de las necesidades especiales (AINSCOW, 2005). El concepto sin embargo se fue ampliando incluso en los sucesivos documentos de la UNESCO y extendió su alcance a todas las personas que por algún motivo, socio-económico, étnico, cultural, de género o de habilidades veían restringido su acceso y permanencia a los distintos niveles del sistema educativo.

En América Latina, la reflexión sobre este concepto tiene características particulares poniendo especial acento en la relación entre la inclusión social y la educación inclusiva. Esta particularidad encuentra origen en el intento de reparar las consecuencias profundamente negativas que las políticas neoliberales de finales de siglo veinte han tenido sobre las sociedades latinoamericanas en general y sobre sus sistemas educativos en particular (RAMBLA et al, 2008). Es decir que la educación inclusiva en el contexto latinoamericano está especialmente vinculada a la inclusión social, sin resignar otras difereficias que pueden generar restricciones al acceso y permanencia de las personas 
dentro del sistema educativo. Hay desigualdades sociales, territoriales, y diferencias de capital simbólico (BOURDIEU, 1991) que hacen que para algunos sectores de la población no puedan acceder a los niveles superiores del sistema educativo incluso frente a la ausencias de restricciones formales para el ingreso como en el caso argentino.

En este artículo adoptaremos la visión más amplia que asume a la educación inclusiva como una corriente dentro del pensamiento de la educación que busca eliminar restricciones y estimular el acceso y permanencia en el sistema educativo a grupos o sectores sociales que por motivos de diferencia social, étnica, cultural, racial, de género, o habilidades han visto restringido o negado su acceso y permanencia dentro del sistema educativo. Por encontrarnos dentro del contexto latinoamericano, nuestro abordaje le dará especial atención a la cuestión de inclusión social por eso utilizamos el concepto "socio-inclusivo" reiteradamente.

Por último este estudio y la experiencia que es objeto del mismo, se refiere a la educación superior inclusiva, es decir que está centrado en los niveles más altos del sistema educativo, particularmente en la educación universitaria. La educación superior suscita gran interés en esta discusión, puesto que la universidad es un lugar, donde se suelen replicar/amplificar las segregaciones y desigualdades sociales que operan en otros ámbitos sociales (BRITO et al,2019). Pero además introduce la cuestión del acceso al conocimiento científico que se produce y enseña en las universidades y el acceso a los beneficios que produce esta forma de conocimiento.

\section{METODOLOGÍA}

La metodología principal para elaborar este estudio fue el análisis documental que consiste en reunir, seleccionar y analizar datos que están en forma de "documentos" producidos por la sociedad para estudiar un fenómeno determinado. Se entiende por documentos los soportes materiales que contienen información y cuya finalidad es la de transportar información y hacer que ésta sea accesible (SANS, 1999). Consiste entonces en el análisis del soporte material de hechos, fenómenos y manifestaciones de la realidad social, que existe con independencia de la acción del investigador. Los documentos son de sumo5interés en la investigación social pues además de construir el marco referencial 
teórico, permiten conocer aspectos históricos, contextuales (demográficos, situacionales, etc.), normativos, organizacionales, institucionales, de opinión pública, entre otros, relacionados con el objeto de investigación (BARRANCO EXPOSITO, 2001).

En particular algunos de los documentos relevantes, entre otras fuentes consultadas, fueron los producidos por la UNaHur donde manifiesta sus objetivos y diseña sus políticas, así como aquellos generados por la institución donde registra el sector social, cultural, nivel educativo, historia familiar de los estudiantes que concurren a la misma.

\section{LA UNIVERSIDAD EN ARGENTINA: ¿PARA QUIÉNES Y PARA QUÉ?}

A lo largo de la historia argentina, la formación de sujetos pedagógicos acordes al modelo de país deseado por los gobiernos que alternadamente tomaron el Estado, ha sido una constante.

En este sentido, la universidad en la Argentina, desde su creación hasta la actualidad, ha expresado la visión del Estado que la materializa. ¿Para qué se piensa la universidad en cada etapa histórica, y quiénes están invitados a participar de ella? Realizaremos un recorrido cronológico intentando responder a este interrogante.

La primera universidad radicada en el territorio que posteriormente sería parte de la Argentina es la Universidad de Córdoba, una casa de altos estudios fundada por la orden jesuita en el año 1613. El proceso de creación de instituciones educativas a partir de la conquista de América forma parte de un segundo momento de intento de "civilizar" el entorno dominado: la evangelización primero, la educación después. Las instituciones políticas y educativas fueron los centros desde los cuales cristalizar el orden y subordinación al poder real y religioso. La ciudad de Córdoba poseía una gran importancia por ser un lugar estratégico de paso intermedio en el camino comercial que llevaba de Buenos Aires a Potosí, en una lógica de organización económica del espacio virreinal. La universidad será el espacio de formación de una elite letrada que formará parte de una burocracia destinada a jerarquizar y reorganizar el espacio colonial primero, y el independentista después. La distancia en el derecho a la "palabra escrita" que se presenta en el interior de la sociedad americana establece un adentro y un afuera: la restricción a la difusißn del aprendizaje de la lectura y la escritura creó un "exclusivismo" que sacralizó la 
letra y a quienes podían ejecutarla (RAMA, 1998). La atención puesta en la formación de letrados universitarios no tuvo ningún paralelo en la creación de un sistema de educación en primeras letras. Adicionalmente debemos tener en cuenta que en América no existía nobleza, por lo cual la posesión de un título universitario constituía una forma de consagración de una burguesía en búsqueda de consolidación. Cuando en 1821 se crea la Universidad de Buenos Aires, como parte de las reformas ejecutadas por Bernardino Rivadavia, en un intento de modernización de la que había sido la capital de la revolución, que incluyó también un Colegio de Ciencias Morales y de Agricultura (TERNAVASIO, 1995). La necesidad de suplantar las antiguas instituciones coloniales (y sus autoridades) por cuadros profesionales burocrático-liberales, generará la fundación de este espacio de estudios, para producir los profesionales necesarios tanto para la vida privada, como para integrar la administración del estado naciente y también formar a los "doctores" que debían gobernar (UNZUÉ, 2012). Estas serán las dos universidades existentes el territorio posteriormente argentino hasta finalizar casi el siglo XIX. La universidad será arancelada, sujeta a exámenes de ingreso de acuerdo a la carrera elegida, y por tanto restringida a grupos que ocupaban las altas esferas de la sociedad decimonónica.

En 1885, ya con un sistema de educación popular pensado para la alfabetización de la población infantil, se dictó la Ley Avellaneda en un acuerdo social que pensó la universidad como un espacio de formación de profesionales (PUIGGRÓS, 2018). Lo interesante es detenernos un momento en qué tipo de profesionales, y para qué son formados. La función de la universidad no radica en la investigación o expansión científica, sino en la certificación de conocimientos. La universidad será el espacio de estudio de carreras liberales (abogacía, medicina, economía, más adelante agricultura o veterinaria), donde se da fe de la adquisición de saberes necesarios para ejercer la profesión decidida (BUCHBINDER, 2005).

El Estado aseguró, para los egresados de las universidades, el ejercicio profesional, dándoles un lugar central en la sociedad e importantes ingresos económicos. Es por eso que la universidad se transformó en un espacio de enorme utilidad para el ascenso social de quienes pudieran ingresar a ella.

Este modelo universitario produjo numerosos cuestionamientos, especialmente centræddos en la falta de formación científica, y el enorme interés en el progreso material e 
individual de quienes la conformaban, constituyendo un lugar de conservación de los vínculos e influencias intraelite, desde donde se podía llegar con facilidad a los cargos públicos. Las discusiones políticas que en la universidad se producían, quedaban circunscriptas a la lucha facciosa más que acrecentados por el debate de a qué modelo de país debía responder. La creación de la Universidad de la Plata en 1896 responde justamente a ese intento de pluralización profesional, pero no redituó mayores éxitos.

Será Córdoba, ese espacio universitario tan dominado por las elites locales, de dónde surgirán demandas extendidas a todo el continente sobre el ser esencial de la universidad. Estamos hablando del movimiento que dará inicio a la Reforma Universitaria de 1918. Tanto el contexto internacional como el local son fundamentales para entender las demandas que el movimiento suscita: es la hora de la Revolución Rusa y Mexicana, del fin de la Primera Guerra Mundial. Es el momento en que el viejo mundo parece dar signos de caducidad. Es entonces la hora americana.

En Córdoba, el título universitario jugaba un rol central en la construcción de sus elites. Si bien podemos rastrear esta característica en la base de todos los espacios cercanos a las universidades, en Córdoba la antigüedad de la universidad la liga a la reproducción de una elite universitaria y doctoral, que pasa de la universidad a la clase dirigente. Cualquier modificación de la universidad es entonces una modificación de la estructura social (BUCHBINDER, 2018). El reformismo posee un gran elemento de transformación generacional, y diferentes demandas, algunas vinculadas a aspectos éticos y humanos de la formación universitaria bellamente reflejadas en el Manifiesto Liminar "Si no existe una vinculación espiritual entre el que enseña y el que aprende, toda enseñanza es hostil y de consiguiente infecunda. Toda la educación es una obra de amor a los que aprenden." (MANIFIESTO LIMINAR, 1918), al conservadurismo de los claustros universitarios "Las Universidades han llegado a ser así fiel reflejo de estas sociedades decadentes que se empeñan en ofrecer el triste espectáculo de una inmovilidad senil”(MANIFIESTO LIMINAR, 1918), la ausencia de una perspectiva científica en sus objetivos formativos "Por eso es que la ciencia, frente a estas casas mudas y cerradas, pasa silenciosa o entra mutilada y grotesca al servicio burocrático"(MANIFIESTO LIMINAR, 1918), a la imposibilidad de democratización "Nuestro régimen universitario aún el más recier\&e- es anacrónico. Está fundado sobre una especie de derecho divino; el derecho 
divino del profesorado universitario. Se crea a sí mismo. En él nace y enélmuere"(MANIFIESTO LIMINAR, 1918).

La universidad consecuencia de la Reforma tiene grandes logros vinculados con el autogobierno y el nacimiento de una vida política universitaria intensa, al igual que grandes limitaciones, especialmente en el campo de la formación científica, el ingreso a la universidad (que continuó siendo limitado) y una gratuidad que no fue prioritario para el movimiento.

Esa gratuidad llegará a la universidad de la mano del gobierno de Juan Domingo Perón. En 1948 existían en Argentina ocho universidades nacionales. Ese año, se creará la UON (Universidad Obrera Nacional). La creación de este espacio de estudios, tuvo relación directa con un proyecto de país que buscaba transformar la tradicional identidad nacional ligada al agro, en busca de un crecimiento de la matriz industrial, con mano de obra calificada y profesionalizada. La creación de una universidad obrera, expresada de esa forma, es ya una gramática de la rebelión social.

Mediante el decreto 29337/49, el poder ejecutivo suprimió los aranceles universitarios. Meses después, se creó un sistema de becas para apoyar el ingreso y permanencia en la universidad, creando además el CONICET (Consejo Nacional de Investigaciones Técnicas y Científicas). Mediante la ley 13031, por primera vez los objetivos y funciones del sistema universitario son incorporados en la organización del país, destacando la imprescindible necesidad de formar ciudadanos responsables y comprometidos. Es un modelo de universidad nacional y popular, de desarrollo estratégico (SILEONI, 2018). Las medidas tomadas por el peronismo permiten el incremento del estudiantado universitario de 40 mil a 140 mil estudiantes.

\section{BUENOS AIRES Y SUS UNIVERSIDADES}

El crecimiento de las instituciones universitarias comenzó en el año 1968. Si para ese momento existían 20 universidades nacionales, para 1989 habrá 27 y diez años después 36 (CHIROLEU 2018). La vuelta de la democracia generó la reapertura de la vida política universitaria, la reapertura de la Universidad Nacional de Luján (cerrada por la dictadura milita९), y el aumento de la presencia femenina en los claustros. Pero será a partir de 1990 
cuando comience una expansión de las universidades nacionales que llevará a que pasen de 29 en ese año, a 56 en la actualidad.

Durante el gobierno nacional de Carlos Menem, y provincial de Eduardo Duhalde en 1995, se dicta la Ley de Educación Superior. Si bien esta ley reafirma la gratuidad del sistema universitario, deja la puerta abierta para el arancelamiento y acelera la apertura de universidades privadas (PUIGGRÓS, 2018). Serán esos también años de fuerte expansión de las universidades bonaerenses, llegando a 15 las nuevas instituciones en un período de diez años. La prioridad será el asentamiento universitario en partidos del conurbano bonaerense con altos niveles de desigualdad social, en una búsqueda de acercar la oferta académica a espacios alejados de la ciudad de Buenos Aires, que contaba con el monopolio de la educación superior estatal. La cercanía al centro de estudios es un elemento central a tener en cuenta para pensar la inclusión educativa universitaria: llegar a una universidad nacional del centro de la Ciudad de Buenos Aires para alguien que habita la zona oeste del Gran Buenos Aires, implica un promedio de entre una hora y treinta minutos, o dos horas. Estamos hablando de un promedio de tres horas de viaje para ir y volver al lugar de cursada. Esta distancia implica una imposibilidad de inserción en los estudios superiores de una enorme cantidad de personas pertenecientes a los sectores populares, para las que un tiempo de traslado y el costo que insume son incompatibles con su vida laboral y familiar. La proximidad es esencial a la hora de pensar la inclusión en el sistema universitario.

En el año 2007, durante la gestión de Néstor Kirchner y Cristina Fernández después, comenzó el debate en el congreso por la nueva ley de Educación Superior. Para 2015, al terminar el gobierno de Fernández, Argentina contaba con 19 nuevas universidades nacionales. El desarrollo de las instituciones se dio en paralelo a políticas públicas de apoyo al estudiantado, como becas de ayuda económico (PROGRESAR), y el Programa Nacional de Becas Bicentenario al que accedieron alrededor del 13\% de los asistentes a instituciones universitarias. El plan de tutorías que consta en el Programa de Calidad Universitaria permite el seguimiento y apoyo a la permanencia dentro de la universidad. Si la anomia propia del sistema es parte de la deserción estudiantil, la generación de tutorías permite el vínculo con el espacio universitario y los docentes que lo forman. El aumento del presupuesto universitario a el 1\% del PBI permitió la continuidad de las políticas públicas en 
educación superior de manera sostenida, y el incremento en estos años de la matrícula universitaria en un 19,7\% (SILEONI, 2018).

El debate en el Congreso de la Nación para la creación de las últimas universidades nacionales creadas en 2014 fue intenso, en un momento político de marcada polarización entre las tendencias que confluirían en los dos partidos mayoritarios de la Argentina: Frente para la Victoria gobernante y Cambiemos, la fuerza opositora. Es interesante leer los argumentos de los últimos ante la creación de nueve universidades nacionales alegando que son pensados como espacios para el proselitismo y aleccionamiento peronista. Trasuntan un conjunto de lugares habituales como la consideración de la incapacidad de discernimiento de los sectores populares que los haría plausibles de dominación electoral, la cercanía de otros centros universitarios (distantes en algunos casos a más de una hora de viaje) o la falta de necesidad de expandir las universidades a un Gran Buenos Aires que cuenta con otras ofertas de formación profesional, dudando de la calidad de la educación que allí se dictaría.

El 19 de noviembre del 2014 se aprobó en la Cámara de Diputados, la ley 27016 que dio origen a la Universidad Nacional de Hurlingham. Días antes había sido aprobada en la Cámara de Senadores. En agosto de 2015 se adquirieron dos inmuebles destinados a su funcionamiento: uno en la calle Vergara 2222, y otro en Origone 151, ambos en Villa Tesei, una de las localidades que integran el partido de Hurlingham. El 21 de septiembre del 2015 comenzaron las inscripciones para sus carreras. En abril del 2016, 1916 estudiantes comenzaron el primer cuatrimestre en la Universidad Nacional de Hurlingham. En marzo de 2020, ese número había trepado a 13 mil.

\section{PROYECTO POLÍTICO-PEDAGÓGICO INCLUSIVO}

La misión institucional de UNAHUR de "contribuir al desarrollo local y nacional por medio de la distribución equitativa de conocimientos e innovaciones científico tecnológicas [se sustenta en] un fuerte compromiso con la excelencia y la inclusión" (MENDONÇA, 2019, p.66). La perspectiva socio-inclusiva es un factor fundacional de la universidad y guía las decisiones que una institución en desarrollo va tomando. Entendemos la inclusión como un conce $\mathbf{\beta}$ to aplicable, ligado a la territorialidad y a los sujetos a los cuales interpela. En este 
sentido, es necesario caracterizar a la población de la universidad para luego poder presentar herramientas de formación e investigación que se amolden mejor a las necesidades y deseos de estas personas. De este modo, el camino a la inclusión se vuelve genuino y no meramente enunciativo.

En los siguientes apartados se caracterizará el perfil del alumnado de UNaHur, sus demandas y necesidades. Se trata por supuesto de una caracterización que puede abordarse desde diferentes perspectivas. Las formas de conocer a esta población serán determinantes en el diseño de una joven institución que siente la inclusión como norte. En este sentido y luego de caracterizar su perfil socioeducativo general, se hará especial énfasis en la matriz intercultural de la población de la universidad y en la perspectiva de género que la UNaHur ha adoptado. Por último, se desarrollará la propuesta de investigación de la universidad que constituye un pilar central de cualquier casa de estudios superiores y que también se desarrolla con especial atención a la respuesta a demandas de la comunidad de Hurlingham.

\subsection{Una universidad en y para Hurlingham}

La más joven de las universidades del conurbano crece año a año. Lo que empezara en 2015 como un proyecto sobre la base de cuatro institutos (Salud Comunitaria, Educación, Tecnología e Ingeniería y Biotecnología), hoy alberga a más de 13000 estudiantes y casi 500 docentes que se distribuyen en más de veinte carreras. La elección de estos institutos para iniciar sus actividades no es casual. La universidad desde sus inicios buscó responder a las demandas de los vecinos ofreciendo carreras de las áreas en las que la zona presenta déficit de profesionales activos. En el caso de Educación, por ejemplo, se responde a la falta de docentes en las escuelas secundarias del partido a partir del crecimiento de la matrícula con la obligatoriedad del nivel en la Ley de Educación Nacional promulgada en 2006. Por otro lado, la universidad especializa y prestigia a la plantilla docente de Hurlingham al ofrecer un Ciclo de Complementación de dos años que otorga a los docentes el título de Licenciado en Educación.

El acceso a la educación superior es un fenómeno reciente para la población del conu $\mathbb{R}$ ano bonaerense. Las tendencias generales muestran que las universidades 
fundadas en las últimas décadas albergan a más de 90\% de estudiantes de primera generación universitaria. La experiencia de los 13000 estudiantes de UNaHur constituye un hito en sus desarrollos familiares y comunitarios. Por otro lado, la universidad se inscribe en una zona con particulares desafíos en términos socio-inclusivos. El partido presenta un 20,2\% de población con carencias múltiples (SIEMPRO, 2010). Estas personas no solo muestran un ingreso por debajo de la línea de pobreza sino que también presentan problemas en su acceso a la salud y la educación y tienen viviendas precarias.

En un contexto por demás desafiante, la universidad brinda a los habitantes de Hurlingham acceso al derecho a la educación y una posibilidad de mejora en sus condiciones de vida, la de sus familias y las de todo el entorno al dotar al conurbano de profesionales formados. Enpalabras de una estudiante:

\begin{abstract}
Estoy casada y tengo dos hijos. Curso en la Universidad Nacional de Hurlingham mi segundo año de la carrera de Letras. Provengo de una familia de clase baja y el secundario lo cursé con 18 años en un colegio nocturno de Capital, después del trabajo. Durante cuatro años fui abanderada. Volvía a mi casa pasada la medianoche y me levantaba a las seis y media todos los días. Cierto día del 2016 vi un cartel que anunciaba la inauguración de la Universidad de Hurlingham. Había un profesorado de Letras. Un año tardé en animarme. Un problema para mí era la edad: me consideraba vieja. Me preguntaba ¿qué iba a hacer yo ahí entre tantos jóvenes? Un día me decidí. llevé la documentación y me inscribí. Mi familia se enteró después. Tenía que dar este paso sola. Me apoyaron desde el principio y se sintieron orgullosos. Me resulta difícil expresar con palabras lo que significa la universidad para mí. Me siento orgullosa de esta facultad que recibe con tanto amor a tantos y cumple sus sueños. Volver a estudiar para mí fue bueno, pero pertenecer a esta Universidad es grandioso. Silvia Maciel, estudiante de Letras UnaHur (QUERER ES PODER, 2018, p.1)
\end{abstract}

\title{
5.2 El perfil intercultural de UNaHur
}

Como se ha mencionado, la raigambre territorial es uno de los factores formativos de la identidad institucional de la UNaHur. Una universidad que no conozca y accione en función de la realidad del entorno en el que se sitúa difícilmente podrá lograr su objetivo de construir una educación verdaderamente inclusiva. Uno de los factores centrales de esta mirada inclusiva es la adopción de una perspectiva multicultural y multilingüe.

Según datos del Censo Nacional de 2010 (CENSO DEL BICENTENARIO, 2012) el partido de Hurlingham posee 9998 vecinos de origen extranjero sobre una población total 
de 181241 habitantes. Dentro de ese grupo, 7098 provienen de países limítrofes. Este panorama configura al partido como un espacio de migrantes. Si bien la constitución de esta población es variopinta, existe una colectividad que destaca del resto por cuantiosidad: la comunidad paraguaya. Según arroja el último censo, en el partido viven 4237 paraguayos y paraguayas, mayoritariamente ubicados en los barrios 9 de Julio y Roca I. Se trata del principal colectivo migratorio de Hurlingham, seguido por la comunidad boliviana con 959. El resto de los grupos migrantes no llegan a mil habitantes cada uno.

Esta realidad de configuración territorial con un alto porcentaje de migrantes sobretodo provenientes de países como Paraguay y Bolivia que tienen lenguas originarias de América como lenguas nacionales lleva a pensar que la universidad se constituirá como un locus de interacción intercultural y un espacio multilingüe. La lengua oficial de Paraguay, además del español, es el guaraní jopara o paraguayo, hablado por el 90\% de la población paraguaya. Incluso se estima que el 57\% de dicha población es monolingüe en guaraní (MELIA, 2004). El caso de Bolivia es aún más radical en el reconocimiento de su perfil multilingüístico ya que reconoce en la Ley $n^{\circ} 269$ :

\footnotetext{
Son idiomas oficiales del Estado, el castellano y todos los idiomas de las naciones y pueblos indígena originario campesinos, que son el Aymara, Araona, Baure, Bésiro, Canichana, Cavineño, Cayubaba, Chácobo, Chimane, Ese ejja, Guaraní, Guarasu'we, Guarayu, Itonama, Leco, Machajuyai-kallawaya, Machineri, Maropa, Mojeño-Trinitario, Mojeño-Ignaciano, Moré, Mosetén, Movima, Pacawara, Puquina, Quechua, Sirionó, Tacana, Tapiete, Toromona, Uru-Chipaya, Weenhayek, Yaminawa, Yuki, Yuracaré y Zamuco (BOLIVIA, DECRETO SUPREMO, 2015)
}

Para conocer el perfil lingüístico del alumnado, en el marco del PIUNAHUR 2 "Las Gramáticas Argentinas: tensiones de lo singular", se realizó una encuesta sociolingüística a los ingresantes. Coincidentemente con los datos censales, el guaraní se destaca como la principal lengua no europea que los ingresantes manifiestan hablar como lengua segunda, con 48 hablantes sobre 701 encuestados (FRUCTUOSO, CALIFA, 2019). En números mucho más reducidos pero no por eso despreciables, la encuesta releva la presencia de hablantes de otras lenguas americanas como el quichua santiagueño, 6 hablantes, el quechua 4, hablantes y el wichí, 1 hablante.

El panorama multicultural y multilingüe no solo es una realidad en la composición del ahtmnado de la universidad sino que encuentra su correlato en la oferta de materias a 
cursar. Por dar dos ejemplos, el Departamento de Educación ofrece las asignaturas “Pensamiento Pedagógico Latinoamericano" que busca formar a los estudiantes en una reflexión sobre la enseñanza y el aprendizaje anclada en nuestro territorio y "Cultura y Literatura de los Pueblos Originarios de América" que brinda rudimentos de estudios lingüísticos, antropológicos y literarios sobre los pueblos indígenas de Sudamérica.

La articulación de la universidad con el territorio también se evidencia en su perspectiva multicultural y, en especial, en relación a otro nivel formativo con el que la UNaHur acciona desde sus inicios: la escuela secundaria. En 2019 las alumnas de la materia Prácticas Docentes en Contexto Formal de la Carrera de Letras, Adriana Arbuco y Celeste Bois, realizaron actividades sobre leyendas mapuches en la Escuela Secundaria $n^{\circ} 5$ de Hurlingham. A raíz de esta exitosa acción, docentes y estudiantes de la universidad participaron en el acto del Día de la Bandera en el cual se presentaron dos banderas de ceremonias: la argentina y la whipala.

\subsection{El recorrido formativo}

Una vez identificado y caracterizado el perfil del estudiante de UNaHur, la Secretaría Académica se dio a la tarea de diseñar un recorrido formativo inclusivo para estudiantes que en su enorme mayoría se están relacionando no solo ellos sino toda su familia por primera vez con una casa de estudios superiores. Como en la mayoría de las universidades del conurbano, más de tres cuartas partes de los alumnos son de primera generación universitaria. Esta situación requirió el diseño de estrategias ad-hoc como las que se mencionan a continuación.

Los ingresantes a las diferentes carreras deben asistir al Curso de Preparación Universitaria (CPU). Se trata de una serie de cursos que están fundamentalmente pensados para hacer un diagnóstico del estudiantado, lo que luego permitirá un mejor acompañamiento a lo largo de sus estudios (MENDONÇA, 2019, p. 69). El curso no es selectivo ni tiene notas finales pero sí es de cumplimiento obligatorio su cursado. Tiene una duración de ocho semanas y consta de tres talleres: Lectura y Escritura, Pensamiento Matemático y Vida Universitaria. El último de ellos responde directamente a la situación de 
un alumnado de primera generación universitaria al dotarlos de herramientas para un mejor desempeño ante el nuevo desafío que la universidad les presenta.

Además de los talleres obligatorios que conforman el CPU, los estudiantes tienen a su disposición talleres abiertos a los que pueden recurrir en cualquier momento de su trayectoria académica. El Laboratorio de Escrituras, por ejemplo, es un recurso al que se puede acceder para redactar una monografía o leer un texto académico que resulte particularmente complejo.

Por último, la cursada de las distintas materias del grado cuenta con una serie de instancias de evaluación pero también de recuperación. Los parciales de las materias pueden ser recuperados para alcanzar la nota necesaria para la promoción o el acceso a la instancia de examen final. También se cuenta con una evaluación integradora entre la cursada y las fechas de finales para aquellos casos en los que sea necesario revisar algún contenido en específico. Si los alumnos rinden este examen focalizado correctamente, aprueban su materia sin necesidad de rendir el examen final.

Como es evidente, la universidad ha tomado una serie de decisiones pedagógicas pensadas específicamente para la población de la universidad. Con la inclusión educativa como meta pero sin resignar la excelencia académica, se ha dotado al recorrido formativo de los estudiantes de un amplio abanico de recursos que tiendan a facilitar el proceso de aprendizaje.

\subsection{Perspectiva de género}

Un aspecto ineludible al desarrollar una educación inclusiva es la adopción de una perspectiva de género. En esta línea, el Consejo Superior aprobó en 2018 el “Programa de Desarrollo de Políticas Universitarias de Igual de Género”. Este programa promueve la incorporación de la perspectiva de género en la formación de los estudiantes así como también desarrollar proyectos de investigación y extensión universitaria con esta perspectiva.

Se busca con la adopción de este programa integrar la perspectiva de género al Régimen Académico y de Convivencia de la universidad, ampliar la representación de las 
mujeres en la política universitaria, generar proyectos de Educación Sexual Integral (E.S.I.), entre muchas otras líneas.

Como hito fundacional de la adopción de este programa, en mayo de 2019 la Dra. Dora Barrancos, renombrada especialista en estudios de género, dictó una clase abierta sobre el tema para toda la comunidad académica.

\subsection{Investigar en UNAHUR}

La investigación ha ocupado un lugar de privilegio entre los objetivos de desarrollo de la universidad desde su creación. En sus documentos fundacionales, la investigación científica es asumida como una de las funciones sustanciales de la universidad y entendida como construcción de conocimiento colectivo, participativo e interdisciplinario, con una fuerte apuesta a su relevancia y pertinencia en el desarrollo social local y de la región en donde se inserta (ROCA et al, 2018).

La decisión de darle un lugar al desarrollo científico desde los inicios de una joven universidad que debía responder a una demanda en primera instancia de formación de estudiantes de grado fue sin dudas ambiciosa. En palabras del Secretario de Investigación, Dr. Juan Pedroza:

\footnotetext{
Sucedió algo bastante innovador, porque ya en el primer cuatrimestre de la historia de la institución, cuando era necesario cubrir las demandas de tres mil doscientos estudiantes preinscriptos, se decidió empezar a investigar. [...] La UNAHUR, por decisión del rector, avanzó en paralelo con las tres funciones universitarias: docencia, extensión e investigación (AULA ABIERTA, 2019).
}

La perspectiva inclusiva y el abordaje territorialmente situado definieron y definen las agendas de investigación de la universidad. Ya en su primera convocatoria a proyectos de investigación anuales de UNaHur (PIUNAHUR) en 2016 se plantean los siguientes ejes temáticos:

- Impacto de la presencia de UNaHur en la comunidad de Hurlingham.

- Diagnóstico ambiental y social del partido de Hurlingham.

- Radiografía tecnológica del partido de Hurlingham y su zona de influencia.

-17Mapa sanitario del partido de Hurlingham. 
En la siguiente convocatoria, PIUNAHUR 2, del año 2017, se hace patente el interés de la universidad de incluir en sus desarrollos científicos a una institución que, como se ha señalado ya, es central a los objetivos de inclusión y democratización del conocimiento: la escuela secundaria. En esta convocatoria, los veintitrés proyectos aprobados se enmarcaron en el eje: la implicancia y vinculación de la universidad pública en relación con los desafíos que enfrenta la educación secundaria.

En los años sucesivos, las diferentes convocatorias a líneas de financiamiento de proyectos de investigación fueron buscando la vinculación territorial estratégica que apunte al desarrollo y fortalecimiento de investigaciones con perspectiva inclusiva y potencial transformador del entorno con el cual la universidad dialoga y en el cual se inserta. Se trata, sin dudas, de un área pujante de la universidad que ya ha superado su objetivo de llegar a 2020 con veinte investigadores radicados y que busca la conformación de un perfil de investigador joven que pueda desarrollarse en la universidad de la mano de una universidad también joven y que también se encuentra en constante desarrollo.

\section{CONCLUSIONES}

"Los dolores que nos quedan, son las libertades que faltan" Manifiesto Liminar 1918

La democratización del acceso a la educación superior implica ineludiblemente la reducción de las desigualdades sociales y los privilegios antes reservados para un pequeño sector de la sociedad. Pensar la inclusión educativa universitaria únicamente en términos de la multiplicación de edificios que alberguen estudiantes es insuficiente. Permitir el acceso a la universidad es esencial, pero debe estar acompañado por un conjunto de transformaciones que permitan que ese espacio inaugurado sea plausible de ser habitado.

Este trabajo ha observado que política universitaria y política de inclusión universitaria son dos caminos distantes. Sólo algunos gobiernos nacionales han trabajado en la segunda, y han sido quienes permitieron con sus decisiones el aumento real de las universidades y los estudiantes que las viven. 
Nuestro país posee el mayor número de estudiantes universitarios de América Latina, con porcentajes que doblan a países como México y Brasil (GRIMSOM, 2018). La educación universitaria argentina se ha caracterizado por ser la más inclusiva de América Latina. Mientras que en muchos lugares del mundo las universidades prestigiosas son de gestión privada, en Argentina la universidad pública y gratuita es la depositaria de ese estatus. Este logro no fue azaroso. Las transformaciones que se han dado en nuestro sistema universitario son el fruto de luchas sociales, y fundamentalmente de acciones políticas que permitieron que aquellos derechos que fueron otorgados sean ejercidos. La distancia entre una universidad que preste servicio a la demanda privada, y otra que cumpla con un desarrollo nacional fundamental para la autonomía geopolítica es profunda.

La Universidad Nacional de Hurlingham es una respuesta a las demandas comunitarias, algunas audibles, otras largamente silenciadas y postergadas. Consideramos que una inclusión que se precie de tal debe estar en directa vinculación con el territorio en que esté instaurada, pensada en y para él, buscando, como manifiesta en su carta fundacional la UNaHur, contribuir al desarrollo local y nacional, a través del impulso al conocimiento y la ciencia. La particularidad de poseer un programa multicultural, multilingüe y multietario, reflejado en la fundamentación y matrícula de la universidad, permite el acceso a la instrucción a sectores que de otro modo, habrían visto vedada o aletargada la experiencia de participar de una casa de estudios superiores. Factores como la distancia (física y cultural) de otros espacios universitarios, incomprensible para sectores que tradicionalmente contaron con la universidad como un derecho dado per se, han sido para los sectores populares que en gran medida engrosan la matrícula de la UNaHur, elementos decisivos para negarles el derecho a este nivel educativo. La universidad es un espacio de crecimiento ciudadano. El derecho al conocimiento debe ser siempre el punto de partida desde el que pensar nuestro sistema universitario.

\section{REFERENCIAS}

AINSCOW, Mel. Developing Inclusive Education Systems: What Are The Levers For Change? JournalofEducationalChange, v. 6, n. 2, p. 109-124, 2005. 


\section{REVISTA}

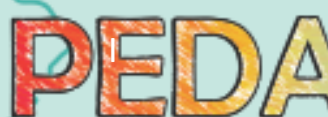

Revista do Programa de Pós-Graduação em Educação da Unochapecó

ISSN 1984-1566 (on-line) ISSN 1415-8175 (impressa)

AULA ABIERTA. Espacio de pensamiento educativo. Investigar desde el día cero: una decisión política. Hurlingham, 2019. Disponible em:

http://aulaabierta.unahur.edu.ar/investigar-desde-el-dia-cero-una-decision-politica/ . Acceso em: 30 jul. 2020.

BARRANCO EXPOSITO, Carmen. Las fuentes documentales en Trabajo Social. Servicios Sociales y Política Social, n. 53, p. 131-149, 2001.

BRITO, Sonia; BASUALDO PORRA, Lorena; REYES OCHOA, Luis. Inclusión Social/Educativa, en Clave de Educación Superior. Revista latinoamericana de educación inclusiva, v.13, n..2, p. 157-172, dic. 2019.

BOLIVIA: Decreto Supremo n 2477, 5 de agosto de 2015. Disponible en: https://www.lexivox.org/norms/BO-DS-N2477.html. Acceso em: 27 jul. 2020.

BOURDIEU, Pierre. La distinción. Criterio y bases sociales del gusto. Madrid: Taurus Humanidades, 1991.

BUCHBINDER, Pablo. El ciclo reformista. In: PERCZYK, Jaime, et al (Org). A cien años de la Reforma Universitaria: Conferencias en la Universidad Nacional de Hurlingham.

Hurlingham: Editorial Libros UNaHur, 2018.

BUCHBINDER, Pablo. Historia de las Universidades Argentinas. Buenos Aires:

Sudamericana, 2005.

CENSO DEL BICENTENARIO. Buenos Aires: INDEC, 2012. Disponible em: https://www.indec.gob.ar/ftp/cuadros/poblacion/censo2010_tomo1.pdf. Acceso em: 29 jul. 2020.

CHIROLEU Adriana. Democratización e Inclusión en la Universidad Argentina durante los gobiernos Kirchner (2003-2015). Educação em Revista, n. 34, p. 1-26, 2018.

FRUCTUOSO, Libertad; CALIFA, Martín. Proyecto PIUNAHUR 02/05. La(s) gramática(s) argentina(s): tensiones de lo singular. Relevamiento sociolingüístico en la Universidad Nacional de Hurlingham. Informe presentado a la Secretaría de Investigación UNAHUR. 2019 .

GRIMSOM, Alejandro. El derecho al conocimiento y el aniversario de la Reforma. In PERCZYK, Jaime, et al (Org). A cien años de la Reforma Universitaria: Conferencias en la Universidad Nacional de Hurlingham. Hurlingham: Editorial LibrosUNaHur, 2018.

MANGAS, Martín; ROVELLI Horacio. El financiamiento de las universidades nacionales: evolución, impacto distributivo y ampliación democrática. Voces en el Fenix, Buenos Aires, 2020. Disponible em: https://www.vocesenelfenix.com/content/el-financiamientode-las-universidades-nacionales-evoluci\%C3\%B3n-impacto-distributivo-y-ampliaci\%C3\%B3. Acce30 em: 30 jul. 2020. 
Revista do Programa de Pós-Graduação em Educação da Unochapecó ISSN 1984-1566 (on-line) ISSN 1415-8175 (impressa)

MANIFIESTO LIMINAR 1918. Disponible em: https://www.unc.edu.ar/sobre-launc/manifiesto-liminar. Acceso em: 30 jul. 2020.

MELIA, Bartolomeu. Las lenguas indígenas en el Paraguay. Una visión desde el Censo 2002. In: ARGENTER, Joan Albert.; MCKENNA BROWN, Robert (Org) Endangered Languages and Linguistic Rights on the Margin of Nations. Batheaston Villa: Foundation of Endangered Languages, 2004.

PUIGGRÓS, Adriana. Universidad y educación pública: una historia de luchas y derechos. In: PERZYK, Jaime, et al. (Org) A cien años de la Reforma Universitaria: Conferencias en la Universidad Nacional de Hurlingham. Hurlingham: Editorial LibrosUNaHur, 2018.

QUERER ES PODER. Diario Página 12. Buenos Aires, 26 de abril de 2018. Disponible em: https://www.pagina12.com.ar/110661-querer-es-poder. Acceso em: 27 jul. 2020.

RAMA, Ángel. La Ciudad Letrada. Montevideo: Ed Arca, 1998.

RAMBLA, Xavier; FERRER, Ferran; TARABINI, Aina; VERGER, Verger. La educación inclusiva frente a las desigualdades sociales: un estado de la cuestión y algunas reflexiones geográficas. Perspectivas, v. XXXVIII, n. 1, p. 81-96, marzo, 2008.

SANS, Carme. Terminología sobre Treball Social i Serveis Socials. Barcelona: Universidad de Barcelona, 2000.

SIEMPRO - SISTEMA DE INFORMACIÓN, EVALUACIÓN Y MONITOREO DE PROGRAMAS SOCIALES. Informe del Partido de Hurlingham. Buenos Aires: Consejo Nacional de Coordinación de Políticas Sociales, 2019.

SILEONI, Alberto. Honrar aquellos sueños. In: PERZYK, Jaime, et al (Org). A cien años de la Reforma Universitaria: Conferencias en la Universidad Nacional de Hurlingham, Editorial LibrosUNaHur, 2018.

TERNAVASIO, Marcela. Construir poder y dividir poderes. Buenos Aires durante la "feliz experiencia" rivadaviana. Boletín del Instituto de Historia Argentina y Americana "Dr. Emilio Ravignani", Tercera serie, n. 26, pp. 7-43, segundo semestre, 2004.

ROCA, Alejandra; SCHNEIDER, Cecilia; SÁNCHEZ, Adriana; PEDROSA, Juan; CHIAPPE. Diana. Investigación y transferencia en las nuevas universidades del conurbano. En ROCA, Alejandra; SCHNEIDER, Cecilia (Org), El legado reformista en las universidades del conurbano: inclusión, democracia, conocimiento. José C. Paz y Avellaneda: EDUNPAZ/UNDAV, 2018.

UNAHUR. Historia. 2016. Disponible em: http://www.unahur.edu.ar/es/historia. Acceso en: 30 jul. 2020. 
Revista do Programa de Pós-Graduação em Educação da Unochapecó ISSN 1984-1566 (on-line) ISSN 1415-8175 (impressa)

UNZUÉ, Martín. Universidad y desarrollo. Las políticas públicas de Ciencia y Técnica y sus relaciones con la democratización de la investigación. In: MAURO, Sebastián; DEL VALLE, Damián; MONTERO, Federico (Org). Universidad pública y desarrollo: innovación, inclusión y democratización del conocimiento. Buenos Aires: IEC-CONADU-CLACSO, 2015.

Enviado em: 28-05-2021

Aceito em: 25-07-2021

Publicado em: 05-08-2021 